\title{
Describing function of two masses with backlash
}

Fernando B. Duarte $\cdot$ J. Tenreiro Machado

\begin{abstract}
This paper analyzes the dynamical proper- ties of systems with backlash and impact phenomena based on the describing function method. It is shown that this type of nonlinearity can be analyzed in the perspective of the fractional calculus theory. The fractional dynamics is compared with that of standard models.
\end{abstract}

\section{Keywords}

Describing function - Backlash · Impacts ·

Control $\cdot$ Modelling $\cdot$ Power law $\cdot$ Fractional calculus

\section{Introduction}

Backlash is one of the most important nonlinearities that taxes the control strategies implemented in the mechanical systems and degrades the overall performance of the systems. It causes delays, oscillations, and consequently gives rise to inaccuracies in the position and velocity of the system. In extreme cases, backlash related effects can help set in an extremely complicated system behavior thereby making it completely intractable from the point of view of the controller. The control of systems with backlash has been investigated by several researchers. In [4, 11-13] the authors considered this problem and developed an algorithm for the compensation of kinematic backlash based on an adaptive controller. This kind of nonlinear dynamic phenomenon has been an active area of research but well established conclusions are still lacking, mainly due to the considerable randomness and diversity of reasons underlying the dynamic effects $[7,10]$.

This paper investigates the dynamics of systems that contain backlash and impacts through the describing function (DF) method. The article is organized as follows. Sections 2 and 3 analyze the describing function of mechanical systems with backlash and the results of numerical simulations using the DF, respectively. The existence of power law relationship between several variables typical of systems with fractional calculus is shown. Finally, Sect. 4 draws the main conclusions and addresses perspectives towards future developments.

\section{Mechanical systems with dynamic backlash}

In this section we use the DF method to analyze a system consisting of two masses subjected to dynamic backlash (Fig. 1). 


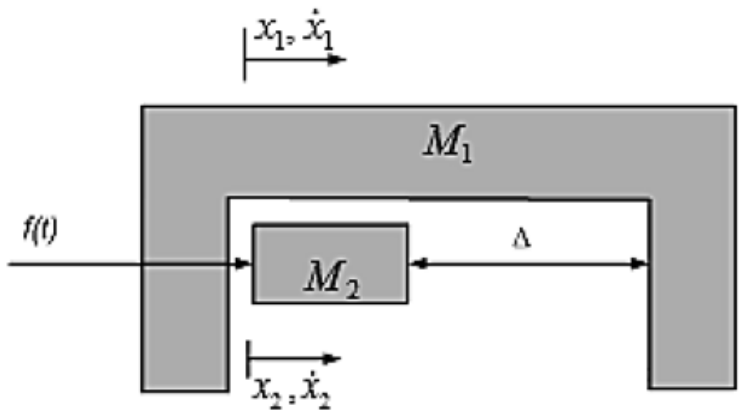

Fig. 1 System with two masses subjected to dynamic backlash

We start, in Sect. 2.1, by considering the standard static model. After that, in Sect. 2.2, we study the dynamical case involving the impact phenomena.

\subsection{Static backlash}

We begin by considering the phenomena of clearance without the effect of the impacts, which is usually called static backlash. The model and its input-output characteristic are shown in Fig. 2. By applying a sinusoidal signal $x(t)=X \sin (\omega t)$ at the input member, the DF of the static backlash is given by the expression [8]:

$$
\begin{aligned}
& N(X)= \begin{cases}0, & X \leq \frac{\Delta}{2}, \\
\frac{k}{2}\left[1-\Psi\left(\frac{X}{1-\frac{X}{4}}\right)\right] & \\
-j \frac{2 k \Delta\left(X-\frac{\Delta}{2}\right)}{\pi X^{2}}, & X>\frac{\Delta}{2},\end{cases} \\
& \Psi(z)=\frac{2}{\pi}\left[\sin ^{-1} \frac{1}{z}+\frac{1}{z} \cos \left(\sin ^{-1} \frac{1}{z}\right)\right] \\
& \text { where } j=\sqrt{-1} \text { and } \Delta \text { is the backlash width. }
\end{aligned}
$$

The classical backlash model corresponds to the DF of a system composed of two masses, $M_{1}$ and $M_{2}$, followed by the geometric backlash, having as input and as output the position variables $x(t)$ and $y(t)$, respectively, as depicted in Fig. 2.

This standard approach to the backlash study is based on the adoption of a kinematic model that neglects the dynamic phenomena involved in the impact process. Because of that, often real results differ significantly from those "predicted" by that model.

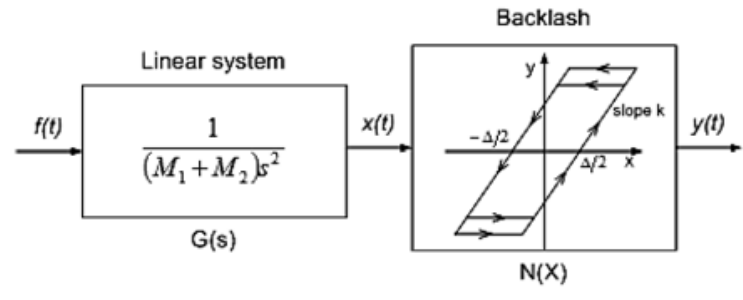

Fig. 2 Classical backlash model

necting their centers of mass. With these restrictions we have velocity components only along this line and no rotational or sliding effects occur. The proposed mechanical model consists on two masses $\left(M_{1}\right.$ and $M_{2}$ ) subjected to backlash and impact phenomenon, as shown in Fig. 1.

A collision between the masses $M_{1}$ and $M_{2}$ occurs when $x_{1}=x_{2}$ or $x_{2}=x_{1}+\Delta$. In this case, we can compute the velocities of masses $M_{1}$ and $M_{2}$ after the impact $\dot{x}_{1}$ and $\dot{x}_{2}$, respectively, by applying the Newton's law:

$$
\dot{x}_{12}^{\prime}=-\epsilon \dot{x}_{12}, \quad 0 \leq \epsilon \leq 1
$$

where $x_{12}=x_{1}-x_{2}$. The coefficient of restitution $E$ varies in the interval $0<E<1, E=0$ being in fully plastic materials and $E=1$ in the elastic ones. By the principle of conservation of momentum it comes:

$$
\begin{aligned}
& M_{1} \dot{x}_{1}^{\prime}+M_{2} \dot{x}_{2}^{\prime}=M_{1} \dot{x}_{1}+M_{2} \dot{x}_{2} . \\
& \text { From (2) and (3) we obtain: } \\
& \dot{x}_{1}^{\prime}=\frac{\dot{x}_{1}\left(M_{1}-\epsilon M_{2}\right)+\dot{x}_{2} M_{2}(\epsilon+1)}{M_{1}+M_{2}}, \\
& \dot{x}_{2}^{\prime}=\frac{\dot{x}_{1} M_{1}(\epsilon+1)+\dot{x}_{2}\left(M_{2}-M_{1} \epsilon\right)}{M_{1}+M_{2}} .
\end{aligned}
$$

From (2) and (3) we obtain:

$$
E_{L}=\frac{\left(1-\epsilon^{2}\right)\left(M_{1} M_{2}\right)}{2\left(M_{1}+M_{2}\right)}\left(\dot{x}_{1}-\dot{x}_{2}\right)^{2} .
$$
by

The energy loss $\left(E_{L}\right)$ at the impact is determined 2.2 Dynamic backlash 
By application of the Newton's law to mass $M_{2}$, we obtain an upper-limit frequency $\omega_{L}$

In this sub-section we consider the case of two bodies colliding on surfaces which are normal to the line con- determined by solving for $x_{2}(t)$ the equation $f(t)=$ $M_{2} x^{\prime \prime}{ }_{2}(t)$ when the amplitude of the displacement is within the clear- ance $\Delta / 2$ yielding [2,3]:

$$
\omega_{L}=\left(\frac{2 F}{\Delta M_{2}}\right)^{\frac{1}{2}} .
$$




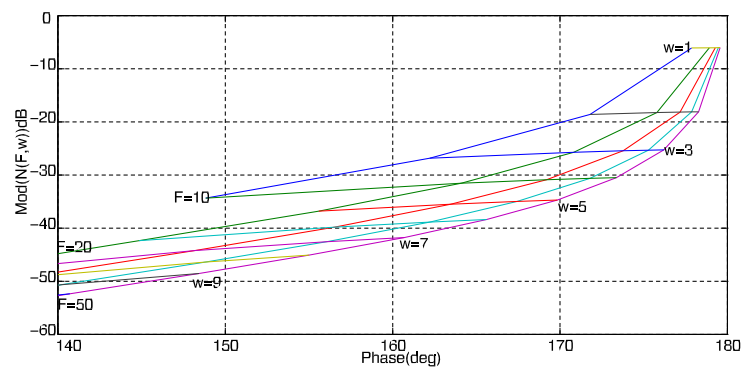

Fig. 3 Nichols plot of $N(F, \omega)$ for the system with static backlash, $10 \leq F \leq 50 \mathrm{~N}, 5 \leq \omega \leq 15 \mathrm{rads}^{-1}$ and $\Delta=0.3 \mathrm{~m}$

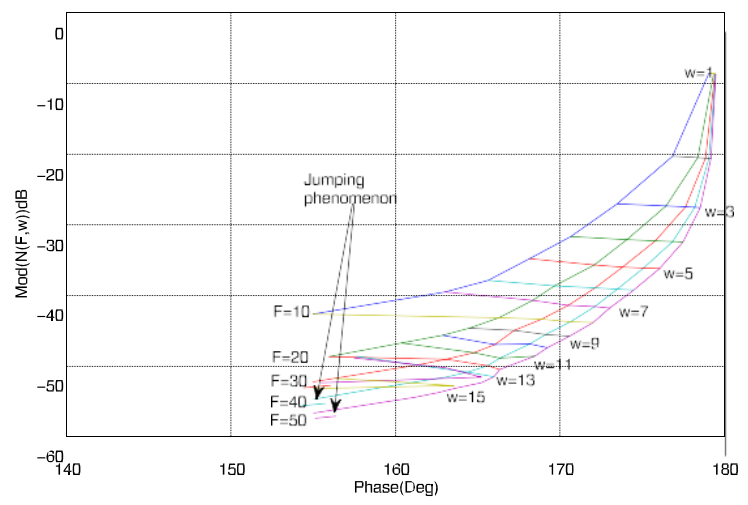

Fig. 4 Nichols plot of $N(F, \omega)$ for the system with dynamic backlash, $10 \leq F \leq 50 \mathrm{~N}, 5 \leq \omega \leq 15 \mathrm{rad} \mathrm{s}^{-1}, \Delta=0.3 \mathrm{~m}$ and $E=0.6$

\section{Numerical simulations}

In this section we study numerically the backlash with impacts.

Figures 3 and 4 show the Nichols plots for $N(F, \omega)$ under the action of an input force $F(t)=$ $F \cos (\omega t)$, with $M_{1}=1.0 \mathrm{~kg}, M_{2}=1.0 \mathrm{~kg}, E=0.6$ and $\Delta=0.3 \mathrm{~m}$, for the static and dynamic backlash models, respectively.

Comparing both charts we note significant differences. Figure 5 illustrates the Bode plots of the static and dynamic backlash models vs. the exciting frequency $\omega$, for the input force $F=40 \mathrm{~N}$. We note that the major difference between the static and dynamic models occurs at the high frequencies. Moreover, Figs. 6-8 show the $\log -\log$ plots of $N(F, \omega)$ with $M_{1}=1.0 \mathrm{~kg}$ and $M_{2}=1.0 \mathrm{~kg}$ for several values of $E$, $\Delta$, and input force $F$. The plots reveal that we have a family of curves that depend on the variation of all the parameters, being more visible for the imaginary component.
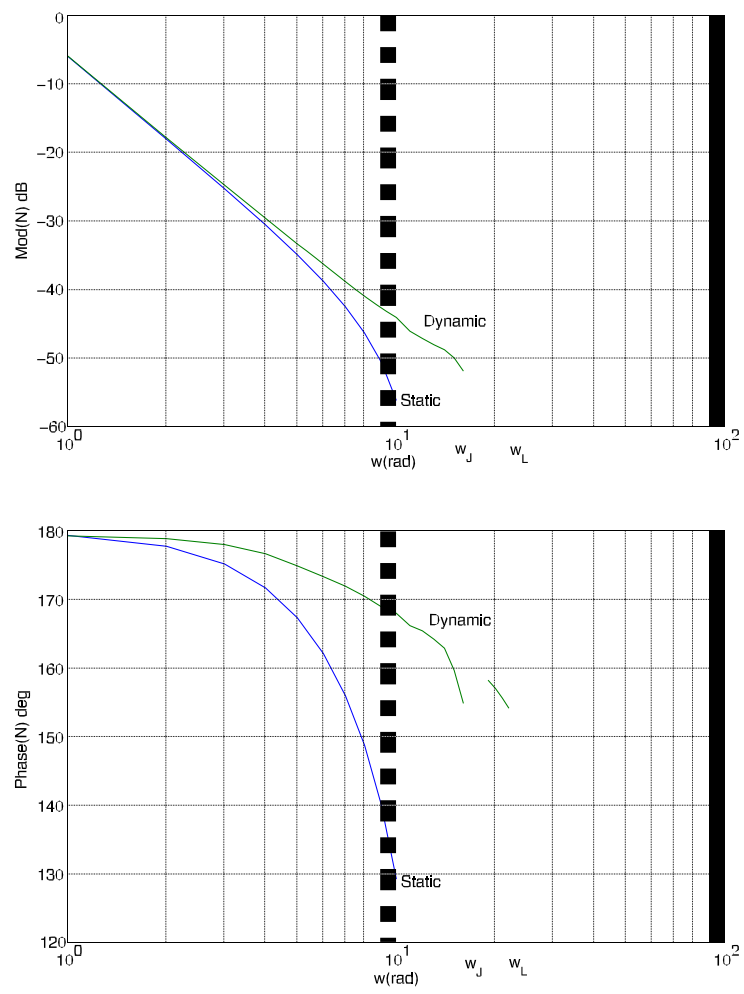

Fig. 5 Bode plots for the magnitude and phase of $N(F, \omega)$ for the system with static and dynamic backlash, $F=40 \mathrm{~N}$, $0.5 \leq \omega \leq 25 \mathrm{rads}^{-1}$ for $\Delta=0.3 \mathrm{~m}$ and $E=0.6$

The Nichols plot in Fig. 4 reveals the occurrence of a jumping phenomenon, $\omega_{J}$, which is a characteristic of nonlinear systems [1]. Moreover, Fig. 4 shows also that for a fixed value of $E$ and $\Delta$ the plots are proportional to the input amplitude $F$. The jumping phenomenon is also visible in Figs. 5-8. Furthermore, it is visible in Figs. 6 and 7, particulary for the imaginary component, that in certain bandwidths the slope that follows is non-integer [5, 6, 9, 14, 15].

The variation of $\omega_{J}$ with $E$ is relatively small. Therefore, in order to study $\omega_{J}$, we consider its average value and we approximate the relationship through the power law function:

$$
\omega_{J} \approx a F^{b}, \quad\{a, b\} \in \mathbb{R} .
$$

Figure 9 illustrates the variation of $\omega_{J}$ versus $F$ for $\Delta=\{0.01,0.025,0.05,0.1,0.2,0.3,0.4,0.5\}$. Figure 10 presents the values of the parameters $\{a, b\}$ versus $\Delta$. We concluded that there is a clear relation- 

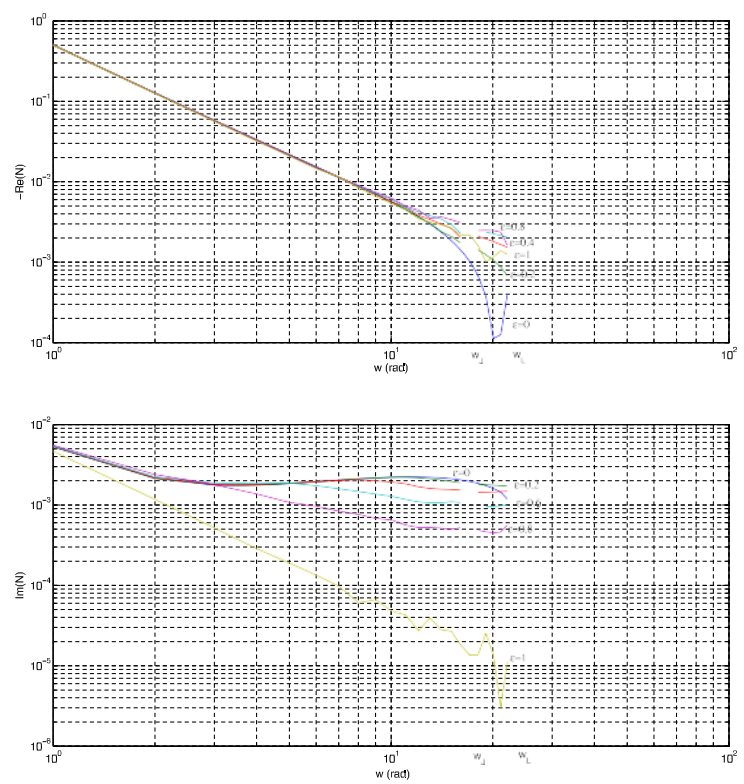

Fig. 6 Log- $\log$ plots of $N(F, \omega)$ for the two mass systems with dynamic backlash, $F=40 \mathrm{~N}, 5 \leq \omega \leq 35 \mathrm{rad} \mathrm{s}^{-1}, \Delta=0.3 \mathrm{~m}$ and $E=\{0.0,0.2,0.4,0.6,0.8,1.0\}$
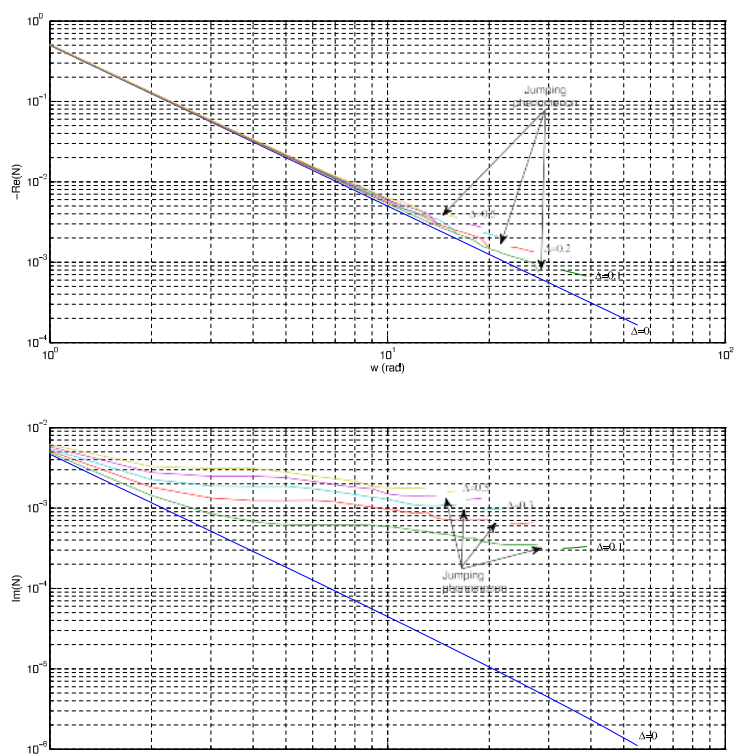

Fig. 7 Log- $\log$ plots of $N(F, \omega)$ for the two mass systems with dynamic backlash, $F=40 \mathrm{~N}, 5 \leq \omega \leq 35 \mathrm{rad} \mathrm{s}^{-1}, E=0.6 \mathrm{~m}$ and $\Delta=\{0.0,0.1,0.2,0.3,0.4,0.5\} \mathrm{m}$

ship between the variables that also approximately follow the power law function:

$$
a \approx 1.193 \Delta^{-0.564}, \quad b \approx 0.565 \Delta^{0.024} .
$$
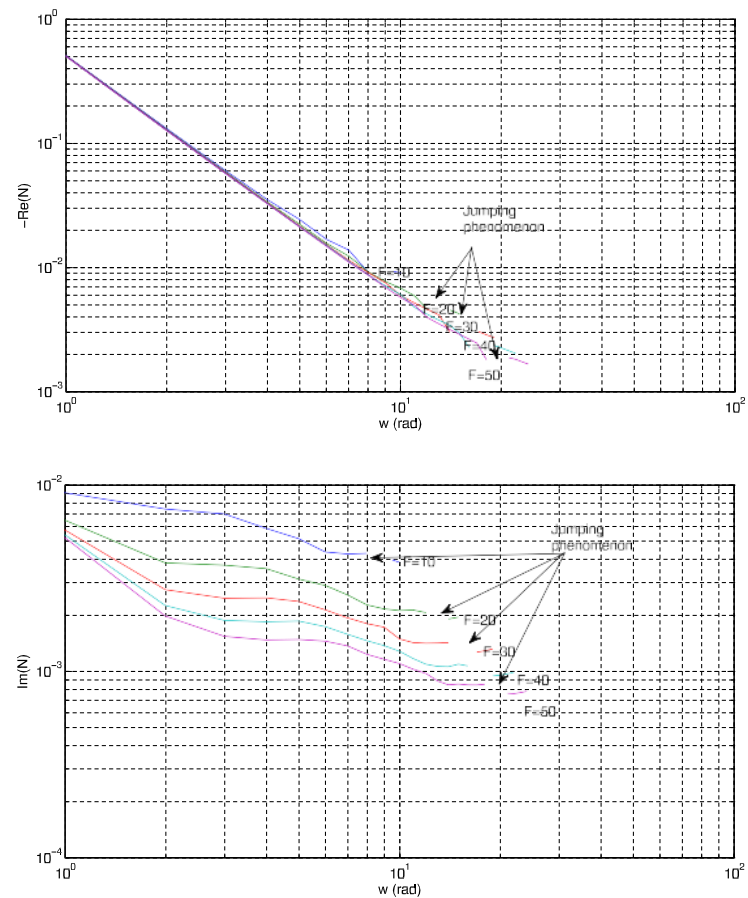

Fig. 8 Log- $\log$ plots of $N(F, \omega)$ for the two mass systems with dynamic backlash, $5 \leq \omega \leq 35 \mathrm{rad} \mathrm{s}^{-1}, \Delta=0.3 \mathrm{~m}, E=0.6$ and $F=\{10.0,20.0,30.0,40.0,50.0\} \mathrm{N}$

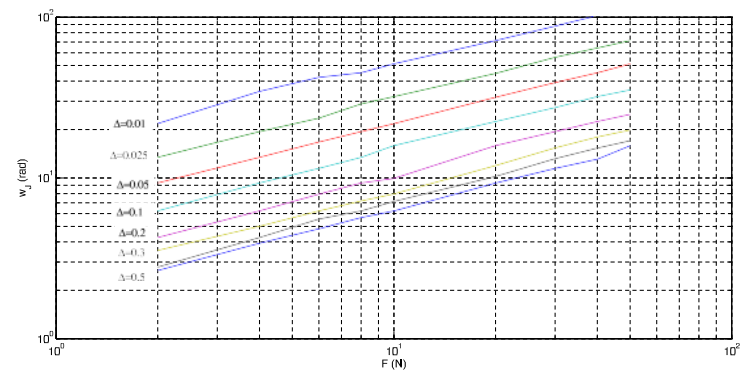

Fig. 9 Variation of $\omega_{J}$ vs. $F$ for $\Delta=\{0.01,0.025,0.05,0.1$, $0.2,0.3,0.4,0.5\}$

Usually it is accepted without a precise mathematical proof that the set of nonlinearities, for which the FD technique can be reliably applied, includes a lowpass filter in the control loop that attenuates the higher order harmonics. Therefore, from an empirical viewpoint, the system with two masses is somehow in the lower limit of applicability of the FD. Nevertheless, experience demonstrates that, in general, the FD is a robust and intuitive scheme leading to responses with a good degree of generalization. 

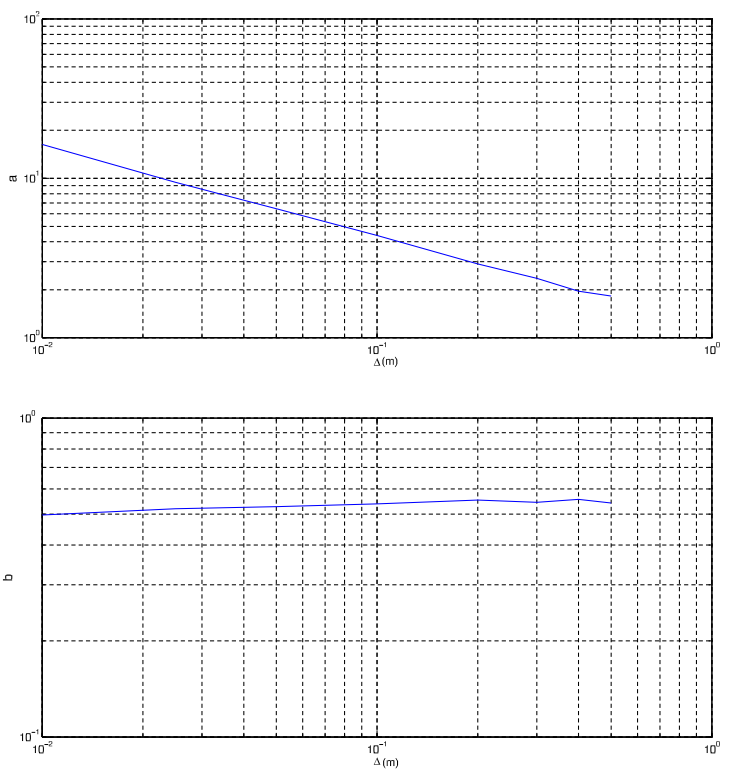

Fig. 10 Variation of the values of the parameters $\{a, b\}$, in $\omega_{J} \approx a F^{b}$, vs. $\Delta$

Another aspect that deserves further comments is the fractional nature of the mathematical description of FD. In general, it is difficult to know a priori if a given phenomenon is of fractional or of integer order. However, a posteriori, usually we can recognize the reasons underlying the fractional dynamics. In the present case we observe that the fractional imaginary component is undoubtedly the description of the multiple dynamic impacts. In fact, without backlash we get a model having a zero imaginary component and with the static backlash we get a $\omega^{-2}$ relationship. Therefore, in these two cases we have integer order dynamics in clear opposition to the fractional dynamics found in the present study.

\section{Conclusions}

This paper addressed several aspects of the phenomena involved in systems with backlash and impacts. The dynamics of a two-mass system was analyzed through the describing function and compared with standard models. The plots revealed that these systems lead to distinct results when adopting static and dynamic models. Furthermore, we observe that several variables follow the power law relationships which were recently recognized to be an important characteristic in nonlinear and fractional dynamical systems.

\section{References}

1. Atherton, D.P.: Nonlinear Control Engineering. Van Nostrand Reinhold Company, London (1975)

2. Azenha, A., Machado, J.A.: On the describing function method and prediction of limit cycles in nonlinear dynamical systems. Syst. Anal. Modell. Simul. 33(3), 307-320 (1998)

3. Barbosa, R., Machado, J.A.: Describing function analysis of systems with impacts and backlash. Nonlinear Dyn. 29(14), 235-250 (2002)

4. Choi, Y.S., Noah, S.T.: Periodic response of a link coupling with clearance. ASME J. Dyn. Syst. Meas. Control 111(2), 253-259 (1989)

5. Duarte, F., Machado, J.A.: Fractional dynamics in the describing function analysis of nonlinear friction. In: 2nd IFAC Workshop on Fractional Differentiation and Its Applications. Porto, Portugal (2006)

6. Lanusse, P., Oustaloup, A.: Windup compensation system for fractional controller. In: First IFAC Workshop on Fractional Differentiation and Its Applications. Bordeaux, France (2004)

7. Leine, R.I., van de Wouw, N.: Stability properties of equilibrium sets of non-linear mechanical systems with dry friction and impact. Nonlinear Dyn. 51(4), 551-583(2008)

8. Phillips, C.L., Harbor, R.D.: Feedback Control Systems. Prentice-Hall, New Jersey (1991)

9. Podlubny, I.: Fractional Differential Equations. Academic Press, San Diego (1999)

10. Slotine, J.E., Li, W.: Applied Nonlinear Control. PrenticeHall, New Jersey (1991)

11. Stepanenko, Y., Sankar, T.S.: Vibro-impact analysis of control systems with mechanical clearance and its application to robotic actuators. ASME J. Dyn. Syst. Meas. Control 108(1), 9-16 (1986)

12. Tao, G., Kokotovic, P.V.: Adaptative control of systems with backlash. Automatica 29(2), 323-335 (1993)

13. Tao, G., Kokotovic, P.V.: Adaptative control of systems with unknown output backlash. IEEE Trans. Autom. Control 40(2), 326-330 (1995)

14. Tenreiro Machado, J.: Analysis and design of fractionalorder digital control systems. J. Syst. Anal. Modell. Simul. 27(2-3), 107-122 (1997)

15. Vinagre, B.M., Monge, C.A.: Reset and fractional integrators in control applications. In: ICCC'2007-8th International Carpathian Control Conference. High Tatras, Slovak Republic (2007) 